\title{
Nanoporous Polymer Films from Immiscible Polymer Blends: Pore Size and Composition Dependence
}

\author{
Tong Liu, Rahmi Ozisik, Richard W. Siegel \\ Department of Materials Science and Engineering, and Rensselaer Nanotechnology Center, \\ Rensselaer Polytechnic Institute, Troy, NY 12180.
}

\begin{abstract}
Nanoporous polymer films have been prepared using immiscible blends of polyetherimide (PEI) and poly(caprolactone diol) (PCLD). The films were prepared by spin-coating from a common solvent - dichloromethane (DCM). To create the nanoporous films, PCLD was removed by immersing the films in acetone, which dissolves PCLD only. Field emission scanning electron microscopy was used to study the porous structure. The pore structure of the films was affected by many factors such as composition of the blend, molecular weight, and various processing parameters. The formation of nanometer size pores was mainly due to the fast phase separation process during spin-coating. The pore size and pore size distribution was found to depend on the blend composition.
\end{abstract}

\section{Introduction}

In recent years, nanoporous materials have been receiving considerable interest from industry because of their several specific characters such as high surface area, low dielectric constant, and low refractive index. Some of the most interesting applications for nanoporous materials are as nanoporous films, which can be used as a catalyst, absorbent, filler system, gas separation layer, or biological sensor. There are many ways to make nanoporous films such as by lithography or the sol-gel method. ${ }^{1}$ One other possible approach is to create a 2-phase mixture and remove the minority phase through physical or chemical means. This is the approach taken in this study through the use of immiscible polymer blends.

Although the preparation of the nanoporous structure is important, a more important issue is the control of the size, distribution and connectivity of the pores within the material. There are many factors that would influence these factors such as polymer blend composition, molecular weight of the blend components, thermal history, and processing conditions. In order to study the effects of these on the final pore structure, a careful characterization of the pores is necessary. Various characterization methods can be used for this purpose, such as mercury injection, gas sorption, permeation test, and optical methods (direct observation). ${ }^{2-5}$ Most of these methods characterize the pore structure by determining various macroscopic properties, which are unique to the pore structure. ${ }^{2}$ Pore structure models are then used to estimate average parameters. However, in the optical methods, pore structure can be directly observed and pore structure parameters can be obtained by image analysis. The disadvantage of these methods is that the statistics is limited due to the small amount of samples (observable pores). To obtain 
global information from the randomly selected regions (which are at the length scales of micrometers or less), statistical methods can be used. ${ }^{6}$

In our study, PEI and PCLD were used to prepare a nanoporous polymer film. The two polymers were dissolved in their common solvent (DCM) and phase separated during spin-coating. Then, the PCLD phase was extracted from the blend by a selective solvent (acetone), thereby creating the pores in the films. The pore structure was characterized by field emission scanning electron microscopy (FESEM). Statistical methods were used to analyze the pore size data based on SEM experiments, and pore structure parameters, such as median pore size and pore size distribution were obtained. This study is focused on characterizing the PCLD content in the blend and its effect on the pore structure.

\section{Experimental and Analysis Methods}

\section{Materials}

PEI (Ultem ${ }^{\circledR} 1000$ ) was obtained from General Electric Plastics, Inc. The number and weight average molecular weight of PEI is 12,000 and $30,000 \mathrm{~g} / \mathrm{mol}$, respectively. The density of PEI is $1.28 \mathrm{~g} / \mathrm{cm}^{3}$. PCLD was purchased from Aldrich Corporation. The number average molecular weight of PCLD is $530 \mathrm{~g} / \mathrm{mol}$ and its density is $1.073 \mathrm{~g} / \mathrm{cm}^{3}$. Dichloromethane (DCM) was used as the common solvent to dissolve both PCLD and PEI to prepare a polymer blend solution for spin-coating. The boiling temperature of $\mathrm{DCM}$ is $40{ }^{\circ} \mathrm{C}$. Acetone was used as the selective solvent to remove the PCLD phase from the films and create the porous structure.

\section{Sample Preparation}

PEI and PCLD were dissolved and mixed in DCM at room temperature using a magnetic stirrer. After the blend solution was formed, films were obtained by adding drops of the solution onto a substrate and spin-coating (SCS P6700 manufactured by Specialty Coating Systems, Inc.). Spin-coating was performed at room temperature and a coating speed of $500 \mathrm{rpm}$ was used. The substrates used in the experiments were highly polished silicon. To create porous films, spun-cast films were immersed in acetone to dissolve and extract the PCLD phase.

\section{Characterization}

In our study, a JEOL JSM-6330F field emission scanning electron microscope (FESEM) was used to study the porous structure of the spun-cast films. The films were first embedded into epoxy resin and a microtome was used to cut the samples. The sample surfaces were sputter-coated with gold in an argon atmosphere.

Image analysis was used to quantitatively determine the pore size and pore size

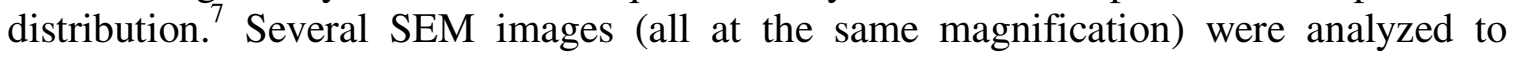
obtain enough data ( $200-300$ pores for each sample) to calculate the median pore size and pore size distribution based on statistical models. We used the probability plot correlation coefficient (PPCC) testing method to both obtain the distribution function and to estimate the distribution parameters. 


\section{Results and Discussions}

Nanoporous polymer films were created by immersing PCLD/PEI blend films in acetone, which dissolved the PCLD phase selectively. The approximate thickness of the films is $1.5 \mu \mathrm{m}$. Figure 1 shows the cross section of a film containing 7\% PCLD (all compositions are based on weight). We observed closed-cell pores (meaning the pores are not connected) at the nanometer length scale. The pores we obtained are nearly spherical in shape. The elongation of some pores in the direction parallel to the film surfaces was due to the deformation during SEM sample preparation. The effective pore size is the average of the longest and shortest diameters of the elongated pores measured from the SEM pictures.

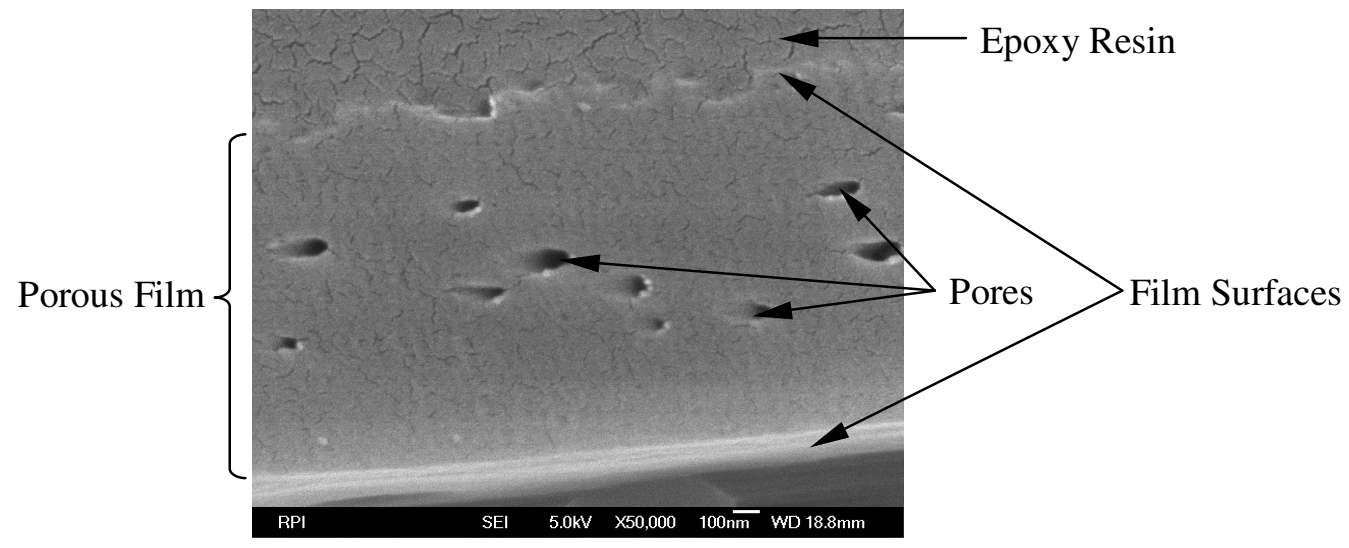

Fig. 1. SEM micrograph of cross-section of a porous PEI film made from a 7\% PCLD/PEI blend.

Pore size is one of the important parameters that describe the pore structure. In our method, the pores were created as a result of phase separation of the two immiscible polymers. The observed pores (and pore size) are directly related to the size of the dispersed domains formed by the minor phase (PCLD), and are controlled by the minor phase content in the polymer blend based on phase coarsening and droplet coalescence phenomena during phase separation process. ${ }^{7}$ Therefore, we used varying PCLD loadings to study the effect of minor phase content on pore size. Pore size distributions were obtained directly by image analysis from SEM micrographs and are shown in Figure 2 for $5,7,10$, and $12 \%$ PCLD content. We counted and classified pores according to their size and calculated the percent of pores. The pore sizes ranged between 50 and $300 \mathrm{~nm}$.

The distributions observed in Figure 2 indicate that there is a greater possibility of having small pores than large pores using this method. In addition, since the pores must always have a finite size $(x>0)$, a lognormal (see Equation 1) distribution is assumed,

$$
f(x)=\frac{1}{x \sigma \sqrt{2 \pi}} \exp \left[-\frac{\ln (x / m)}{2 \sigma^{2}}\right]
$$

where $\sigma$ is the shape parameter, and $m$ is both the scale parameter and the median of the data that is defined as follows:

$$
m=\exp (\widehat{\mu}) \quad \text { and } \quad \hat{\mu}=\frac{\sum_{i} \ln x_{i}}{N}
$$




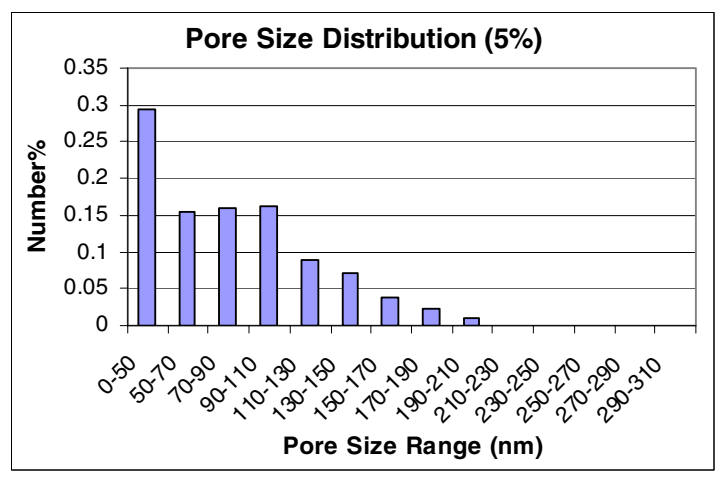

(a)

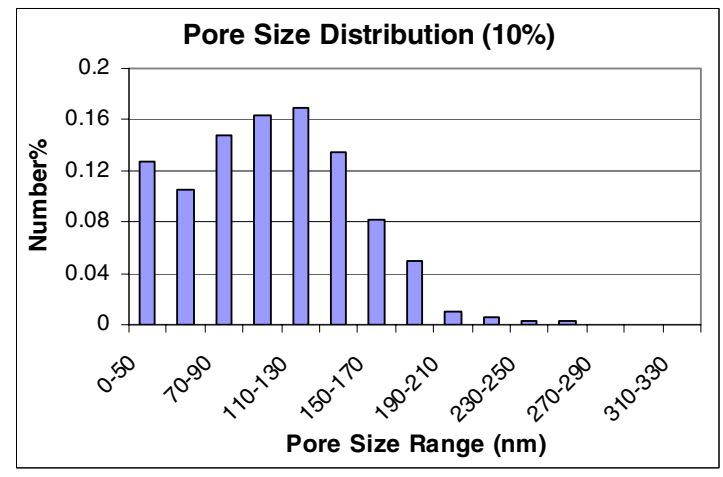

(c)

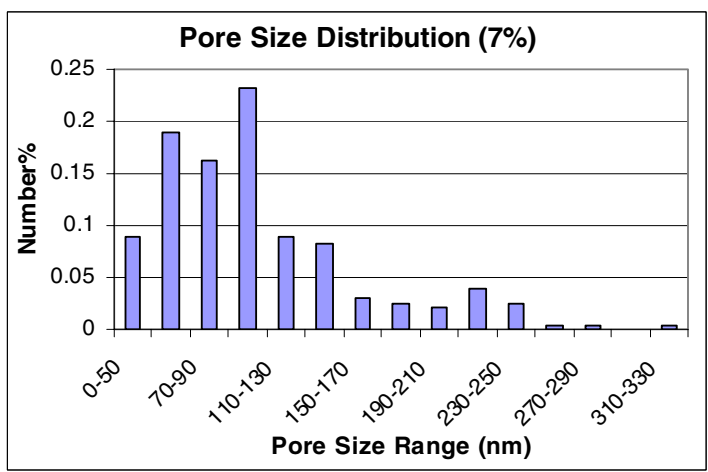

(b)

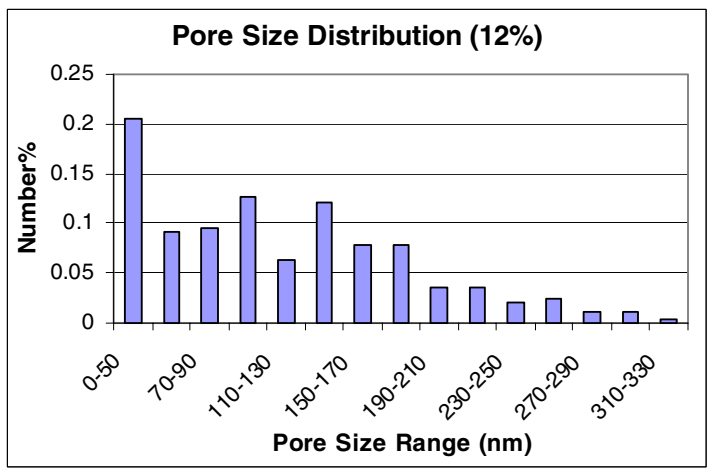

(d)

Fig. 2. Pore size distribution of porous PEI made from PCLD/PEI blends with different PCLD loadings: (a) $5 \%$, (b) $7 \%$, (c) $10 \%$, (d) $12 \%$.

The PPCC method was used to verify whether our data obeys the lognormal distribution function. In the PPCC method, the data can be plotted against an assumed theoretical distribution in a way that the points should follow a linear relationship. ${ }^{8}$ The correlation coefficient associated with the straight line in the probability plot shows the quality of the fit. If the data follow the assumed distribution model, the correlation coefficient would be equal to one. In general, if the correlation coefficient is greater than 0.9 , the distribution function is accepted to represent the data successfully. In addition, the shape and scale parameters of the probability density function can be estimated from the intercept and slope of the linear plot. $^{8}$

Figure 3 shows the pore size distributions of samples with varying PCLD contents and are described by the lognormal distribution function. The correlation coefficient and distribution parameters of the lognormal distribution that were obtained by the PPCC method are given in Table 1. The correlation coefficients for different samples were all close to one, showing that the pore size data can be represented by the lognormal distribution.

Table 1. Correlation coefficient and distribution parameters obtained by the PPCC method.

\begin{tabular}{|l|c|c|c|c|}
\hline PCLD content & $\mathbf{5 \%}$ & $\mathbf{7 \%}$ & $\mathbf{1 0 \%}$ & $\mathbf{1 2 \%}$ \\
\hline Correlation coefficient & 0.985 & 0.996 & 0.981 & 0.976 \\
\hline Median $(\mathrm{m})$ & 74.8 & 94.0 & 99.1 & 103 \\
\hline Shape parameter $(\sigma)$ & 0.489 & 0.487 & 0.407 & 0.583 \\
\hline
\end{tabular}


The median pore size increased with increasing PCLD content (see Figure 4). This is probably due to the increasing coalescence of the minor phase during the phase separation process. The number of minor phase droplets would increase with increasing amount of minor phase that would enhance the probability of aggregation. Thus, larger minor phase domains would form after phase separation and lead to the formation of larger pores after solvent extraction.

\section{Conclusions}

The present study describes a method to create nanoporous polymer films by using immiscible polymer blends and removing the minor phase through solvent extraction. Nanometersize pores were created due to the fast phase separation during the spincoating process. Scanning electron microscopy was used to observe the pore structures. The pores obtained were found to be closed cells. Image analysis was used to measure the sizes of the pores. Statistical methods were used to analyze the data and to obtain the relative information of the pore population. Various samples were prepared with varying PCLD content. It was found that as the PCLD content increased, the pore size also increased. The pore size distribution can be described using a lognormal distribution model. The median pore size increased from 74.5 to $103 \mathrm{~nm}$ as the PCLD content was increased from 5 to $12 \%$ due to increasing coalescence of the PCLD-rich phase during phase separation process.

\section{Acknowledgements}

This work was supported by IBM and the Nanoscale Science and Engineering Initiative of the National Science Foundation under NSF award number DMR-0117792.

\section{References}

1. P. Holister, C. R. Vas, T. Harper, "Nanoporous materials", Technology white paper No. 5, Cientifica Ltd., (2003).

2. F. A. L. Dullien, Porous media, fluid transport and pore structure; Academic: New York, (1992).

3. K. Sakai, K. Ozawa, R. Mimura and H. Ohashi, Journal of Membrane Science 32, 3-17, (1987). 
4. C. Salmas and G. Androutsopoulos, Journal of Colloid and Interface Science 239, 178-189, (2001).

5. Z. Mogri, D. R. Paul, Journal of Membrane Science 175, 253-265, (2000).

6. J. S. Rustagi, Introduction to statistical methods; Rowman \& Allanheld, (1984).

7. C. Chandavasu, M. Xanthos, K. K. Sirkar, C. G. Gogos, Polymer 43, 781-795, (2002).

8. NIST/SEMATECH e-handbook of statistical methods, http://www.itl.nist.gov/ div898/handbook, (2004). 